EXATASE TECNOLÓGICAS

V.3 N.3 • Agosto/Setembro/Outubro - 2019

ISSN Digital: 2359-4942

ISSN Impresso: 2359-4934

DOI: $10.17564 / 2359-4942.2019 v 3 n 3$
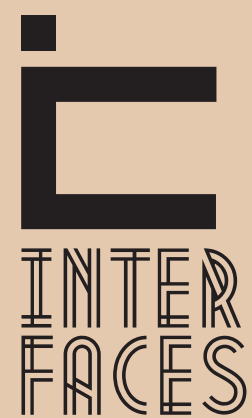

CIENTÍFICAS

\section{COMPREENSÃO DA ELIPSE NA CIRCULARIDADE DAS REPRESENTAÇÕES: EXERCITANDO A CRIATIVIDADE}

UNDERSTANDING OF THE ELLIPSE IN THE CIRCULARITY OF REPRESENTATIONS: EXERCISING CREATIVITY

COMPRENSIÓN DE LA ELIPSE EN LA CIRCULARIDADDE LAS REPRESENTACIONES: EJERCITAR LA CREATIVIDAD

\section{RESUMO}

A Educação Matemática é o espaço da construção da explicação dos objetos da Matemática, mas, na atualidade ainda é ignorada nos espaços de formação e no exercício da docência em Matemática. A elaboração de uma explicação possibilita a construção de significados e caminhos para a descoberta, que são importantes para a Educação Matemática. Uma abordagem semiótica para a Educação Matemática destaca a importância da metodologia da complementaridade das diferentes interpretações sobre um objeto e, por isso, apresentam muitas possibilidades explicativas para construção de significados e caminhos para descoberta. No presente trabalho, perseguiremos caminhos para a compreensão da elipse, por uma construção de significados, elaborando explicações, mudando e criando novas relações para representações geométricas dessa curva, explorando uma circularidade nas representações com utilização de software de geometria dinâmica.

\section{PALAVRAS-CHAVES}

Complementaridade; criatividade; elipse; explicação; geometria dinâmica. 


\section{ABSTRACT}

Mathematics education is the space for the construction of the explanation of the objects of mathematics, but nowadays it is still ignored in the training spaces and the exercise of teaching in mathematics. The elaboration of an explanation enables the construction of meanings and paths for discovery, which is essential for mathematical education. A semiotic approach to mathematics education highlights the importance of the complementarity methodology of the different interpretations of an object and, therefore, presents many explanatory possibilities for the construction of meanings and paths for discovery. In the present work, we will pursue paths for understanding the ellipse, by constructing meanings, elaborating explanations, changing and creating new relationships for geometric representations of this curve, exploring a circularity in representations using dynamic geometry software.

\section{KEYWORDS}

Complementarity. Creativity. Elipse. Explanation. Dynamic Geometry.

\section{RESUMEN}

La educación matemática es el espacio para la construcción de la explicación de los objetos de las matemáticas, pero hoy en día todavía se ignora en los espacios de entrenamiento y en el ejercicio de la enseñanza en matemáticas. La elaboración de una explicación permite la construcción de significados y caminos para el descubrimiento, que son importantes para la educación matemática. Un enfoque semiótico de la educación matemática resalta la importancia de la metodología de complementariedad de las diferentes interpretaciones sobre un objeto y, por lo tanto, presenta muchas posibilidades explicativas para la construcción de significados y caminos para el descubrimiento. En el presente trabajo, vamos a perseguir caminos para entender la elipse, construyendo significados, elaborando explicaciones, cambiando y creando nuevas relaciones para representaciones geométricas de esta curva, explorando una circularidad em las representaciones utilizando software de geometría dinámica.

\section{PALABRAS CLAVE}

Complementariedad; creatividad; elipse; explicación; geometría dinámica. 


\section{INTRODUÇãO}

A motivação para o presente estudo surgiu no exercício da docência no curso de Graduação em Matemática e em formações continuadas de professores, diante da constatação de que alunos em formação inicial na graduação em Matemática e professores da educação básica com licenciatura em Matemática, apresentaram incompreensão de propriedades básicas da elipse, como, por exemplo, a existência de tangentes em cada ponto dessa curva. Essa constatação se revelou em diferentes momentos com diferentes grupos, quando, diante da apresentação de um contorno de uma pista de corrida, Figura 1, construída com duas retas e dois semicírculos foi acrescentada a pergunta: - "essa figura é uma elipse?" - a qual, todos responderam afirmativamente.

A resposta equivocada não foi contestada de imediato, mas, reconduzida para um processo de construção de uma explicação, com a seguinte frase: "certo, se acreditam ser uma elipse, então, peço que provem com quaisquer argumentos que lhes possam parecer significativos para o que estão afirmando". Para contribuir com os argumentos, materiais didáticos foram oferecidos para a construção.

Figura 1 - A falsa elipse

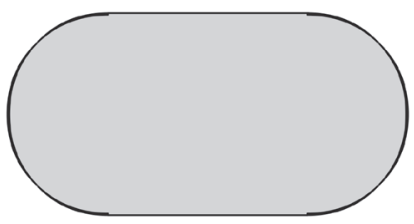

Fonte: Construído no Geogebra pela autora.

Entendemos que a Educação Matemática é o espaço da construção da explicação dos objetos da Matemática, mas, na atualidade vivemos a hegemonia da tendência formalista que prioriza a manipulação simbólica nas estruturas formais da matemática e isso traz dificuldades para a construção de conceitos com significados. Na sistematização da matemática à maneira formalista a explicação poderia se dar apenas por "provas formais" que utilizam o princípio do terceiro excluído, as quais os graduandos ou os já graduados, pouco utilizam, e quando o faz, se limitam a alguma prova reproduzida por memorização, sem construção de significados. Compreendemos que mesmo diante da apresentação de uma prova formal, muitas dificuldades na compreensão permanecem, porque, assim como muitos outros pesquisadores acreditamos que, provas formais não explicam e nada acrescentam além do que já está dito.

Destacamos a importância da explicação no ensino e aprendizagem da Matemática em todos os níveis de ensino, pois, a explicação introduzida aos métodos de construção, ainda é muito ignorada. Steiner (1978) faz a distinção entre diferentes maneiras de provar algo em matemática e destaca que, em vários períodos da elaboração do pensamento matemático encontramos as provas que explicam. Steiner (1978) acrescenta que as provas explicativas por permitirem percepção de objetos geométricos, são instrumentos para a descoberta, também enfatiza a importância que exerce uma proprieda- 
de geométrica visualizável para que aconteçam os processos heurísticos, que são os que chamamos de caminhos para a descoberta.

Observaremos ainda, a importância dos processos interpretativos na Educação Matemática. Em Otte (2012) olhar significa interpretar e para uma abordagem semiótica para a Matemática, entendemos que interpretar pode levar a conceitualizar. Portanto, uma interpretação de um fenômeno, é, também, um processo de criar uma interpretação da interpretação dada e assim por diante. Assim, atuamos na possibilidade da semiose como processo explicativo para um objeto da Matemática.

Otte (1993) descreve o processo criativo como uma operação na interação entre variação e repetição. Para Monteiro (2015), buscar complementaridade entre sentidos e significados na circularidade das representações de um mesmo objeto, torna-se um método para construção de processos criativos no sentido dado por Otte (1993).

Uma epistemologia para a educação matemática, em uma perspectiva semiótica pode ser perseguida por uma metodologia da complementaridade entre sentidos e significados ${ }^{2}$, entre algo, um signo $^{3}$ que cabe na estrutura interpretativa do sujeito, que vai se aproximando de uma semântica quase individual, admitindo diferentes formas interpretativas dentro de sistemas de símbolos e, principalmente, da produção de novos símbolos. Para a produção desses novos símbolos, Monteiro (2015) sugere uma circularidade nas representações dos objetos da matemática.

Por isso, destacamos a importância da exploração das representações geométricas encontradas na literatura tradicional, em diferentes matemáticas, interpretadas em softwares de geometria dinâmica. Nesse intuito, buscaremos apresentar uma possibilidade dessa metodologia da complementaridade entre sentidos e significados orientada por uma circularidade, usando geometria dinâmica para reinterpretar uma percepção sobre a elipse.

\section{METODOLOGIA}

Uma contribuição importante de Steiner (1978) é a distinção entre as provas que apenas provam e as provas que explicam, presentes na Matemática.

Segundo Steiner (1978) para explicar a existência de uma entidade, deve-se deduzi-la por meio da essência ou natureza dessa entidade. Por essência compreendemos as propriedades características de tais entidades; esse autor esclarece:

2 Kant relaciona sentindo (intensão) e significado (extensão) aos termos “ideia” e “objeto, respectivamente. Ele formula essa descrição utilizando os termos "juízos analíticos e juízos sintéticos, e declara "em todos os juízos, nos quais se pensa a relação entre um sujeito e um predicado [...] essa relação é possível de dois modos. Ou o predicado $B$ pertence ao sujeito $A$ como algo que está contido (implicitamente) nesse conceito $A$, ou B está totalmente fora do conceito $A$, embora em ligação com ele. No primeiro caso chamo de juízo analítico, no segundo, sintético" (CRP, A/67 B/10). Kant prossegue explicando: "Os primeiros poderiam igualmente denominar-se juízos explicativos; os segundos, juízos extensivos[...]” (CRP, A/7 B/11). Para Quine (2011), sentido é conotação e significado é denotação.

3 Para Santaella (1995), um signo é “algo", pode ser um gesto, uma marca, um desenho ou uma teoria. São infinitas as possibilidades de se representar uma ideia, gerar um signo. 
Minha proposta é que chamemos de uma prova explicativa aquela que faz referência a uma propriedade característica de uma entidade ou de uma estrutura mencionada no teorema, de tal forma que da prova seja evidente que o resultado depende de tal propriedade. (STEINER, 1978, p. 143).

Outra sugestão de Steiner (1978, p. 146) é que as

[...] provas explicativas poderiam ter sido usadas para descobrir o resultado, e com frequência ocorre o caso de que a prova característica é intuitiva o suficiente para servir como um instrumento de descoberta. Finalmente, uma propriedade característica é como ser visualizável (como certamente é o caso de uma propriedade geométrica) 4 .

A Educação Matemática é o espaço para a construção de um discurso sobre a existência dos objetos da Matemática e suas relações. Perceber e explicar são as faces do problema que temos em mãos. Exploraremos diferentes maneiras de perceber a elipse, estimulando a observação para interpretação. Otte (2012, p. 78), argumenta que

A percepção realmente é um processo construtivo [...] mesmo a percepção sendo uma construção, as condições dessa construção são dadas pelo ambiente e pelas experiências da pessoa. A percepção, dessa maneira, fornece uma hipótese sobre o que a gente está vendo, e esta hipótese tem de ser testada novamente [...].

Ao mesmo tempo, como sugere Monteiro (2015), ao considerarmos a dinâmica nas mudanças que podem sofrer um signo, propomos metodologias que tratem a complementaridade em uma circularidade das interpretações como provocação para a mudança e produção de novos signos. Com apoio das ferramentas tecnológicas, no caso específico aqui, um software de geometria dinâmica, caminharemos por um exercício para criatividade: interpretando, combinando, mudando e criando algo novo.

Assim, esse processo para a construção de significados com complementaridade nas interpretações da elipse foi: 1) iniciado com dobraduras, depois, interpretamos as dobraduras com ajuda de um desenho geométrico, em seguida, combinamos essas maneiras de perceber a elipse e a reinterpretamos em um software de Geometria Dinâmica, e assim, criar outras possibilidades de interpretação. Ao final, descobrimos um caminho para a definição analítica da elipse, encontramos outra maneira para iniciar as dobraduras, abrindo caminho para a criação de outros signos. 0 processo foi iniciado pelas orientações da apostila abaixo, Figura 2.

\footnotetext{
4 Our account of mathematical explanation suggests why the other criteria are plausible. First, generality is often necessary for capturing the essence of a particular, and the same goes for abstraction. [] Another suggestion was that the explanatory proof could have been used to discover the result, and it is often the case that a characterizing proof is intuitive enough to serve as an instrument of discovery. Finally, a characterizing property is likely to be visualizable (as is certainly the case with a geometrical property).
} 
Figura 2 - Material de referência para construção da elipse por dobragem

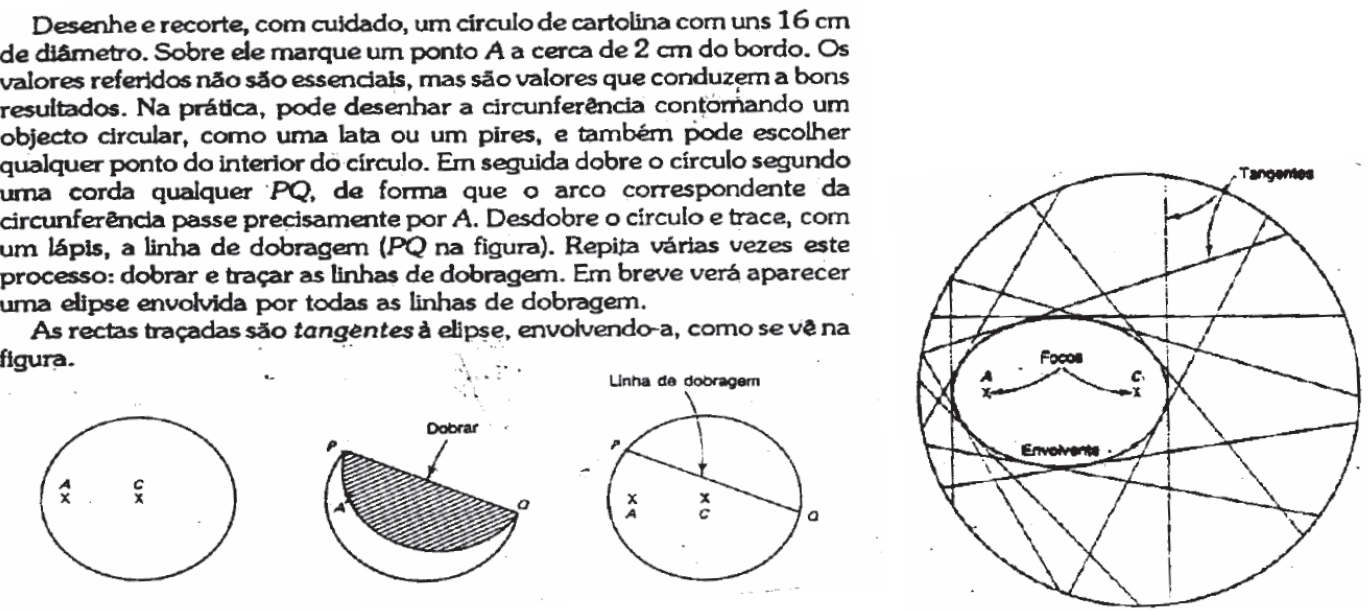

Fonte: Bolt (1990, p. 30).

Depois da experiência por dobraduras os alunos da Licenciatura em Matemática, não questionaram se, de fato, essas dobraduras teriam gerado uma elipse. Então, o processo foi reconduzido por um questionamento - “As dobraduras realmente determinam uma elipse?", para continuarmos às buscas por explicações.

Nessa etapa, o desenho geométrico da Figura 3 foi apresentado como parte desse processo de elaboração. Comparando essas duas representações, os segmentos revelados com as dobraduras, supostas tangentes e o desenho geométrico da Figura 3, foi possível chegar a interpretação de que o círculo que iniciou a oficina das dobraduras, poderia ser um dos círculos diretores da elipse.

Figura 3 - Os círculos principais da elipse

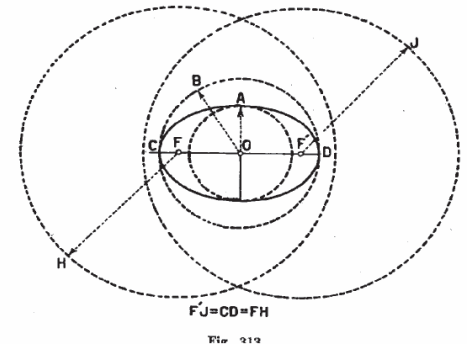

Cículos principais são aquêles traçados com raio igual aos semi- eixos maior e menor da elipse e centro no centro da curva ou seja com raio igual a $O A$ e $O B$ da figura 313 .

Assim temos o círcula principal maior e menor segundo o raio seja o semi-eixo maior ou o menor.

Circulos diretores são aqueles tragados tendo como centro um dos focos da elipse e com raio igual no seu eixo maior, e podem ser vistos na figura com raios $F H$ e $F^{\prime} J$.

$$
F^{\prime} J=C D=F H
$$

Fonte: Carvalho (1969, p. 219).

Comparar a atividade das dobraduras com o diagrama da Figura 3 possibilitou a formulação de duas interpretações com apelo à percepção: primeira, que o círculo usado nas dobraduras seria um 
dos círculos diretores, e, segunda, a percepção de que o ponto escolhido aleatoriamente dentro do círculo para iniciar as dobraduras, poderia ser o outro foco da elipse.

Nesse ponto da análise foi possível elaborar estratégias para construção da elipse em um software de Geometria dinâmica.

Figura 4 - Passo a passo para a interpretação das dobraduras Geogebra, que culmina em um diagrama que relaciona a soma das distâncias dos focos a um ponto da elipse por semelhança de triângulos

$4 a$

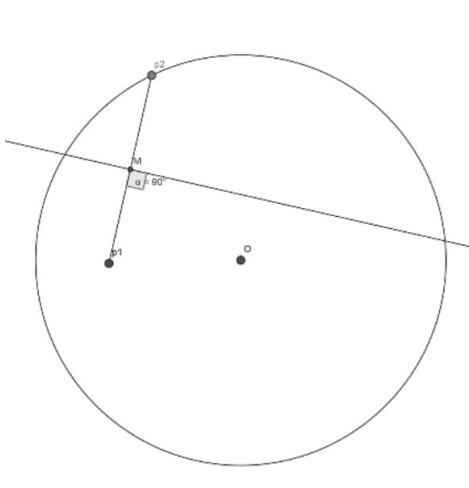

$4 b$

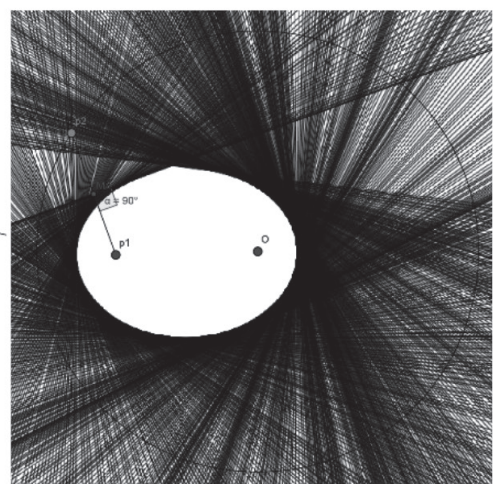

$4 c$

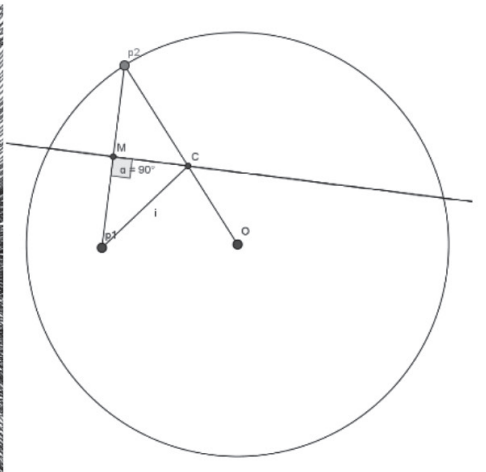

Fonte: Construção no Geogebra pela autora.

As observações no software de geometria dinâmica possibilitou as elaborações subsequentes: perceber que o segmento, na figura 4 a, distância do ponto escolhido na borda com o do interior do círculo, que fizemos coincidir, executando as dobraduras, é intersectado perpendicularmente no ponto médio $\mathbf{M}$ de pela tangente revelada nessa dobradura. Parecia existir elementos suficientes para uma explicação sobre o fato daquelas dobraduras determinarem uma elipse. Com esses recursos foram possíveis outras observações, como por exemplo, determinada uma "dobradura" e, usando a ferramenta "habilitar o rastro" dessa reta, aparecem as incontáveis tangentes e estas coincidem com os pontos de uma elipse revelada, representada na Figura $4 \mathrm{~b}$.

Mas, uma interpretação detalhada das relações entre esses diagramas nessa construção revela, também, uma semelhança entre triângulos muito importante para nossa análise, no diagrama explicativo da Figura 4c. Os triângulos $P 2 M C$ e $P 1 M C$ são congruentes, pois, como a tangente à elipse corta $\overline{P 1 P 2}$ perpendicularmente, no ponto médio, temos:

1) $\overline{P 1 M}=\overline{P 2 M}$

2) Os ângulos definidos pela intersecção entre a reta da dobradura e o segmento $\overline{P 1 P 2}$ medem $90^{\circ}$; pois se intersectam perpendicularmente.

3)O segmento $\overline{M C}$ é comum aos dois triângulos;

Isso caracteriza um caso LAL - “lado, ângulo lado’, de congruência de triângulos, da geometria 
plana e agora temos uma explicação, uma descrição que estabelece novas relações significativas.

Partindo dessa congruência, afirmamos agora que os segmentos $\overline{P 1 C}$, e, $\overline{P 2 C}$ têm a mesma medida. $O$ segmento $\overline{P 2 C}$ somado ao segmento $\overline{C O}$, corresponde ao raio do círculo diretor. Mas, o segmento $\overline{P 1 C}$, que é igual ao segmento $\overline{P 2 C}$, somado ao segmento $\overline{C O}$ também tem a mesma medida do raio do círculo diretor. Agora fica mais evidente que temos uma elipse e que: os pontos $P 1$ e $O$, são os focos e $C$ um ponto genérico da elipse.

Temos o suficiente para formular uma nova descrição para elipse: A soma das distâncias dos focos a um ponto da elipse é igual ao raio do círculo diretor, uma constante procurada e citada na definição analítica ${ }^{5}$. Da definição analítica essa constante é o eixo maior da elipse que é igual ao raio do círculo diretor. Uma nova relação está explicitada, ou seja, uma prova explicativa de que as tangentes das dobraduras, definem uma elipse. Essa interpretação não impede a definição à maneira da geometria analítica, organizada a partir da noção de distância entre pontos, Figura $5 b$.

Figura 5 - Definição da elipse no desenho geométrico e na geometria analítica, respectivamente

(a)

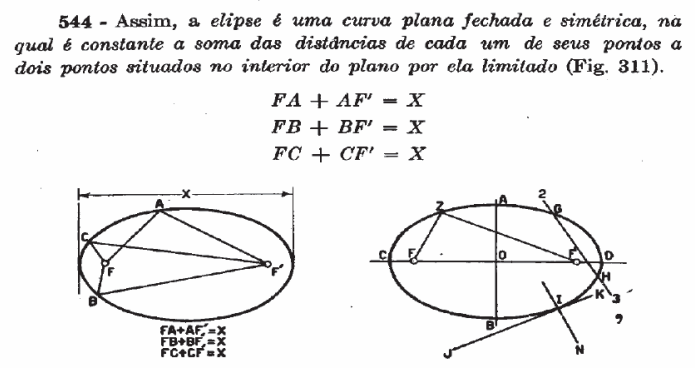

(b)

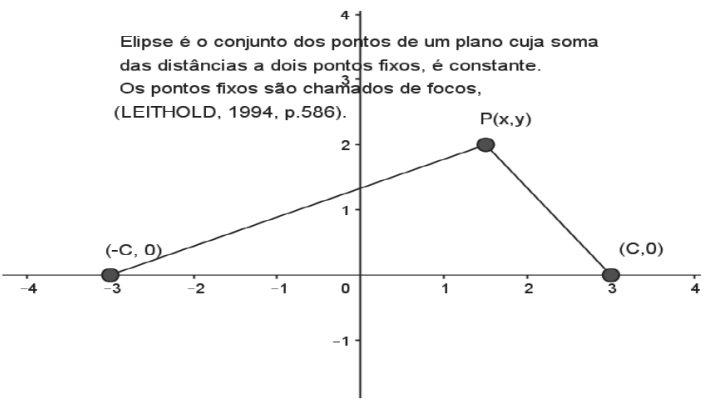

Fontes: 5(a) Carvalho (1969, p. 218); 5(b) Construção no Geogebra.

A explicação de uma verdade matemática pelo método da complementaridade entre os sentidos e os significados, envolvendo software de geometria dinâmica e ideias matemáticas é uma realidade para produção de signos que precisa ser incorporada aos processos de ensino e aprendizagem da matemática, inclusive por ser linguagem significativa na atualidade. Nessa perspectiva, estimulamos a capacidade de interpretação, argumentação, estimulamos a construção de uma explicação, partindo da percepção e alguns conceitos. Esse procedimento traz, também, possibilidades para análise com números, que é linguagem para a matemática e a exploração de explicações, utilizando os objetos construídos na "janela da álgebra”, durante as construções geométricas nesse software.

5 Elipse é o conjunto dos pontos de um plano cuja soma das distâncias a dois pontos fixos, é constante. Os pontos fixos são chamados de focos (LEITHOLD, 1994, p. 586). 


\section{RESULTADOS E ANÁLISE}

Essa maneira de apresentar um estudo sobre a elipse, utilizando combinações de representações, além de exercitar a criatividade, partindo da observação em dobraduras, pode conduzir a criação de novos signos. Depois das relações percebidas anteriormente, surgiram outras possibilidades de produzir outras "oficinas" com dobraduras à elipse, agora com essas novas relações percebidas que sugerem outros passos inclusive para iniciar as dobraduras.

A complementaridade na circularidade, metodologia que aplicamos nas formações de professores de Matemática, como a apresentada nesse artigo, evidencia novas perspectivas para tratamento de curvas tão importantes para o desenvolvimento da Matemática e com vasto material produzido, como por exemplo, a parábola. Em outra experiência para a construção de uma explicação sobre a parábola, investigamos alguns materiais didáticos já produzidos por outros autores, mas, no caso da parábola, iniciamos a observação, com o software de geometria dinâmica, utilizando atividades propostas por Nóbriga (2010), conduzindo as análises para a construção de explicações sobre equações e funções, com outros materiais, passando por um estudo das propriedades características da parábola, como foco e diretriz.

Percebemos que a combinação de material didático disponível, com apoio da ferramenta da geometria dinâmica, constitui-se um campo para exploração do desenvolvimento de conceitos matemáticos por um exercício de criatividade, ou seja, combinando, mudando e criando algo novo. Conduzido por uma metodologia da complementaridade em uma circularidade, esse exercício para a criatividade é também um exercício de interpretação de outras interpretações encontradas que, combinadas com as técnicas disponíveis na cultura digital, gera uma perspectiva diferenciada para a Didática da Matemática.

\section{CONCLUSÃO}

Para Otte (2016), a importância da filosofia para a educação matemática resulta não apenas do fato do conceito de "explicação" ser central para nossas práticas e objetivos educacionais. Mas, para esse autor, a matemática não poderia ser frutuosamente organizada e perseguida na escola se fosse tratada como um tópico primariamente profissional ou como uma mera linguagem sem conteúdo.

Ao tentar educar a geração mais jovem dentro da "sociedade do conhecimento" tecnológica atual, vale a pena lembrar que o conhecimento, quando considerado de um ponto de vista educacional, cumpre dois papéis principais na sociedade humana: técnica e filosófica. A educação geral deve basear-se em conhecimentos científicos comprovados, pelo menos porque "parece que a ciência surgiu com a exigência da [...] coerência e que uma das funções que desempenha permanentemente na cultura humana consiste em unificar [...] As habilidades práticas e as crenças cosmológicas, a episteme e a techne [...] apesar de todas as mudanças que a ciência poderia ter sofrido, essa é sua função 
permanente e específica que a diferencia de outros produtos da atividade intelectual humana “. (AMSTERDAMSKI, 1975, p. 43-44 apud OTTE, apresentação oral, Universidade de Bielefeld, julho de 2016).

Assim, compreendemos que no desenvolvimento histórico do conhecimento matemático, a matemática segue um processo de produção de signos, sem fim. Sugerimos perseguir, na educação matemática, esses passos da matemática assim como o de todas as ciências em busca da complementaridade entre sentidos e significados. A produção de signos na atualidade está contemplada com as possibilidades da interpretação do pensamento matemático na cultura digital, que pode ser explorada como um caminho para muitas descobertas.

\section{REFERÊNCIAS}

BOLT, B. Atividades matemáticas. São Paulo: Gradiva, 1990.

CARVALHO, B. de A. Desenho geométrico. Rio de Janeiro: Ao livro técnico, 1969.

KANT, I. Crítica da razão pura. Trad. e notas Fernando C. Mattos. 2. ed. Petrópolis, RJ: Vozes; Bragança Paulista, SP: Editora Universitária São Francisco, 2013.

LEITHOLD, L. 0 cálculo com geometria analítica. 3. ed. São Paulo: Harbra, 1994.

MONTEIRO, L. C. S. Sentidos e significados para uma abordagem semiótica em educação

matemática: uma análise sobre as discussões das interpretações do paradoxo de Zenão. 2015. 180f. Tese (Doutorado) - Programa de Pós-graduação em Educação Matemática, Universidade Anhanguera de São Paulo - UNIAN, São Paulo, 2015.

MONTEIRO, L. C. S. Paradoxo de Zenão: perspectivas para a educação matemática na interpretação da variação do problema. Encontro Nacional de Educação Matemática, 11. Anais, Curitiba, PR, 2013.

NÓBRIGA, J. C. C.; ARAÚJO, L. C. L. Aprendendo matemática com o geoGebra. São Paulo: Exato, 2010.

OTTE, M. 0 formal, o social e o subjetivo: uma introdução à filosofia e à didárica da matemática. São Paulo: Unesp, 1993.

OTTE, M. A realidade das Ideias: uma perspectiva epistemológica para a Educação Matemática. Cuiabá, MT: EdUFMT, 2012. 
OTTE, M. A filosofia da educação matemática entre o platonismo e o computador. Apresentação oral, Universidade de Bielefeld, Alemanha, julho de 2016.

QUINE, W.V. De um ponto de vista lógico. São Paulo: Unesp, 2011.

SANTAELLA, L. A teoria Geral dos signos: semiose e autogeração. São Paulo, SP: Ática, 1995.

STEINER, M. Mathematical explanation, philosophical studies. D. Reidel Publishing Company, n. 34, p. 135-151, 1978.
Recebido em: 30 de Março de 2018

Avaliado em: 5 de Maio de 2018

Aceito em: 10 de Agosto de 2018

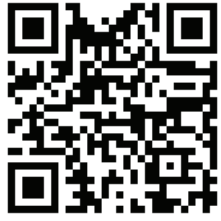

A autenticidade desse artigo pode ser conferida no site https://periodicos. set.edu.br
1 Doutora em Educação Matemática pela Universidade Anhanguera de São Paulo; Mestra em Psicologia Cognitiva pela Universidade Federal de Pernambuco - UFPE; Graduada em Matemática pela Universidade Federal de Alagoas - UFAL; Professora da Universidade Federal de Alagoas - UFAL. E-mail: lucia.monteiro@cedu.ufal.br; http:// lattes.cnpq.br/7348098729151581

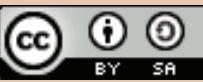

Este artigo é licenciado na modalidade acesso abertosob a Atribuição-Compartilhalgual CC BY-SA

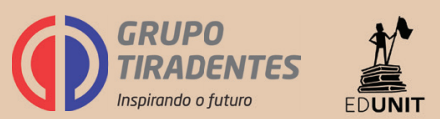


